\title{
N86-19913 \\ Wet-Oxidation Waste Management System for CELSS
}

\author{
Yukio Takahashi \\ Department of Civil Engineering \\ Niıgata University \\ Nigata, Japan \\ Haruhiko Ohya \\ Department of Chemical Engineering \\ Yokohama Natıonal University \\ Yokohama, Japan
}

\section{ABSTRACT}

A wet oxidation system will be useful in CELSS as a facility to treat organic wastes srid to redistribute inorganic cumpounds and elements. However at rather higher temperatures needed in this reaction, for instance, at $260{ }^{\circ} \mathrm{C}$, only $80 \%$ of organic carbon in a raw rivaterial can be oxidized, and $20 \%$ of it will remain in the liquid mainly as acetic acid, which is virtually noncombustible. Furthermore, $n i t r o g e n$ is transformed to ammonium lons which normally cannot be absorbed by plants. To resolve these problems, it becomes necessary to use catalysts. Noble metals such $3 s$ Ru, Rh and so on have proved to be Fartially effective as these catalysts. That is, oxidation does not occur completely, and the unexpected denitrification, instead of the expected nitrification, occurs. So,it is essential to develop the catalysts which are able to realize the complete oxidation and the nitrification.

MANY RESEARCH EFFORTS have been funded to make wet oxidation systems commercially available for the purpose of treating organic wastes, particularly sewage sludge, and since 1957, the Zimmermann Process (Zimpro) has been successfully used in the USA, Japan and other countries. This system seems useful in a Closed Ecological Life Support System (CELSS) to treat and reduce organic components of wastewater from plants, human beings and so on, into inorganic compounds (and elements).

But it seems that the discussions on the applicability of wet oxidation to CELSS does not fully reflect the experlences and results obtained in this field. This paper will discuss: the usefulness and the unsolved problems of wet oxidation in CELSS on the basis of authors' ten-year research 'or wet oxidation of sewage sludge.

\section{EXPERIMENTAL METHODS}

Wet oxidation is a reaction in which organic or reductive compounds are oxidized in the presence of liquid water. This reaction occurs between $100^{\circ} \mathrm{C}$ and $374^{\circ} \mathrm{C}$ which is the critical temperature of water, so the reaction should be carried out in a pressure vessel (autoclave) to prevent evaporation of water.

All data shown later are the results of autoclave tests. The pressure in the autoclave at a given reaction temperature is determined as a sum of the pressures of water and gases which are introduced 1 rito the reactor at a room temperature before the beginning of the experiment. Therefore, it is impossible to keep the reaction pressure below a saturated vapor pressure of water at the given reaction temperature.

Before an experiment, it is necessary to introduce enough oxygen gas into the reactor so as to accomplish the intended oxidation. The rate of oxygen gas in the reactor to the chemical oxygen demand 
(COD) of a raw materi ân oxygen iridex(R)". "In the case of $R=1$, it means that the exact quantity of oxygen has been added to oxidize the raw materials comoletely.

Pure oxygen gas or a mixture of oxygen and nitrogen gases has been used. Even after the determination of " $R$ ", it is possible to exchange the gas ratio of oxygen to nitrogen at will. You rave only to increase or decrease the 'olume of the raw material. Sewage sludge is used as a raw material.

\section{EFFECTS OF THE REACTION TEMPERATURE}

CARBON - Fig.1 shows the relation between the reaction temperature and total organic carbon(TOC). The temperature is increased from $110^{\circ} \mathrm{C}$ to $310^{\circ} \mathrm{C}$ at an interval of $50^{\circ} \mathrm{C}$. Pressures are all set at $75 \mathrm{kgf} / \mathrm{cm}^{\wedge} 2$ except in the case of 310 ${ }^{\circ} \mathrm{C}$, where the pressure is controlled at $115 \mathrm{kgf} / \mathrm{cm}^{\wedge} 2$. The reason why high pressures are applied at lower temperatures of 110 ${ }^{\circ} \mathrm{C}$ or $160^{\circ} \mathrm{C}$ will be discussed later. The reaction time, after the temperature of the reactor reached the designed temperature, is $30 \mathrm{minutes.} \mathrm{Added} \mathrm{oxygen}$ index(R) is unity and pure oxygen is used as an oxygen source.

Total organic carbon[TOC(mix)],

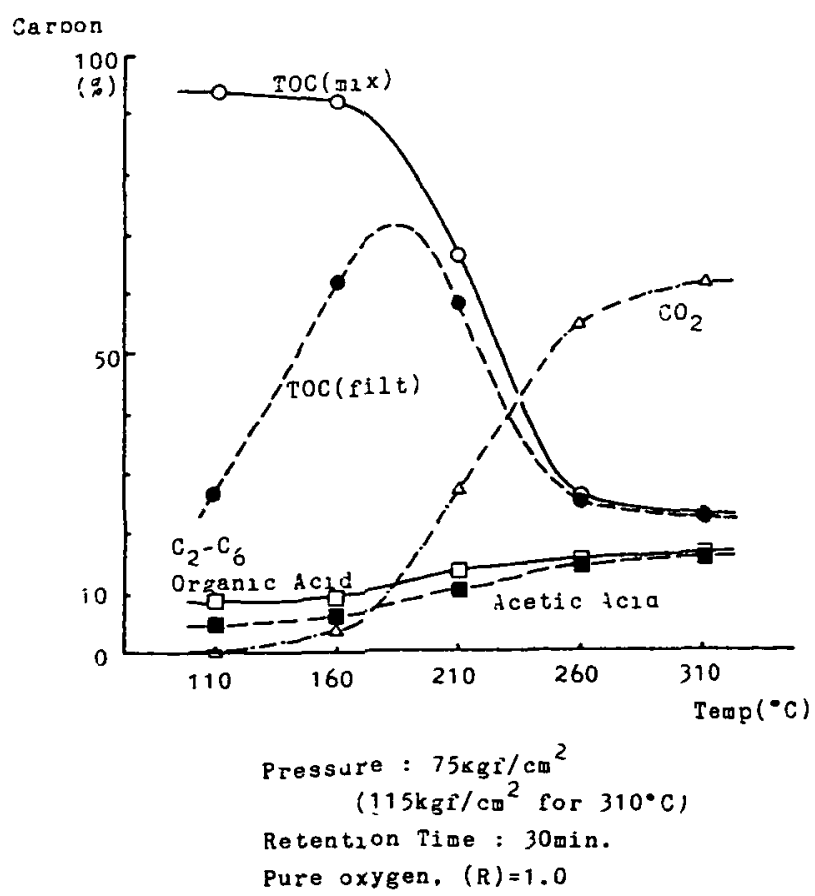

Fig.1 Reaction Temperature and TOC filtrable organic carbon[TOC(fi|t)] and converted, carbon quantities of acetic acid, $C_{2}-\dot{C}_{6}$ organic acidslthe sum of acetic, propionic, l-butyric, n-butyric, i-valeric, n-valer $1 c, 1$-capric, n-capric acids) in oxidlzed liguors are shown in fig. 1 as the percentage of carbon quantities of the raw material versus the respective temperature.

The behaviour of TOC $(\mathrm{mix})$ curves in the figure shows that the oxidation occurs when the temperature is higher than $160^{\circ} \mathrm{C}$, arid this process becomes more active at the higher temperature. However when the temperature exceeds $260^{\circ} \mathrm{C}$, the reaction will not proceed any more. The value of TOC(filt) increases with the increase of temperature and passes through the highest value in the range of $160{ }^{\circ} \mathrm{C}$ to $210{ }^{\circ} \mathrm{C}$, then it decreases anc! approaches to the curve of $\operatorname{TOC}\left(m_{1} x\right)$ after $210^{\circ} \mathrm{C}$.

In the case where the raw material is sewage sludge, solubilization of suspended organic compounds occurs first at a temperature less than $160{ }^{\circ} \mathrm{C}$, ther both solubilization and oxidation proceea in between $160^{\circ} \mathrm{C}$ and $210^{\circ} \mathrm{C}$, and after $210^{\circ} \mathrm{C}$ oxidation only occurs.

With the increase of reaction temperature, amount of $C_{2}-C_{6}$ organic acids increases and their 6 quantity reaches two-thirds of $\operatorname{TOC}(\mathrm{mix})$ at temperatures higher than $260^{\circ} \mathrm{C}$. On the other hand, the ratio of acetic acid in $\mathrm{C}_{2}-\mathrm{C}_{6}$ organic acids increases with the increase of the reaction temperature. When it reaches $260{ }^{\circ} \mathrm{C}$, acetic acid occupies nearly the total amount of $\mathrm{C}_{2}-\mathrm{C}_{6}$ organic acids, and maintains this amount independently to the increase of temperature thereafter.

This does not mean that the reaction had reached equilibrium. Acetıc acid, whose concentration ranges from zero to $16 \mathrm{~g} / 1$ as converted carbon quantities, is not oxidized at all, even if excessive oxygen is added to the reactor. Therefore the main products in wet oxidation is "acetic acid" in addition to carbon dioxide and water.

NITROGEN - Fig.2 shows the relation

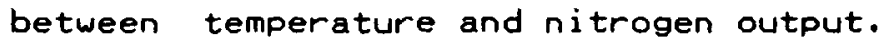
As the temperature becomes higher, the solubilization of suspended nitrogen compounds occurs, and it is almost 
completed at $160^{\circ} \mathrm{C}$. But this solubilization does not resilt in the formation of ammonia. Between $160^{\circ} \mathrm{C}$ and $210^{\circ} \mathrm{C}$, at which both the solubilization and the oxidation of organic compounds occur, production of ammonia increases rapidly. Even if the solubilization has been completed, solutile nitrogen compounds do not become ammorila totally at $210{ }^{\circ} \mathrm{C}$, but at $260^{\circ} \mathrm{C}$.

In wet oxidation without catalysts, the nitrogen compounds may not be oxidized to nitrite and nitrate. On the other hand, forms of nitrogen usable by plants are nitrite and/or nitrate with the exception of some plants such as rie. So in CELSS, it is impossible to apply oxidized liquor directly to plant culture in hydroponic solutions. It is

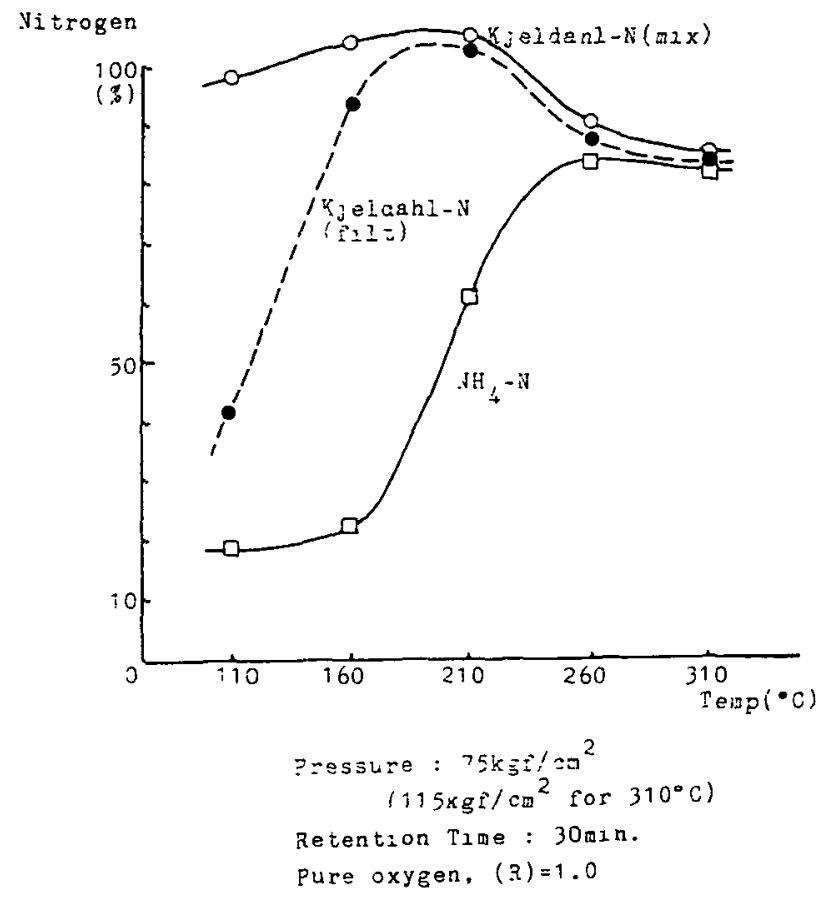

Fig.2 Reaction Temperature and $\mathrm{Nitrogen}$

necessary to convert ammonia to the forms nitrite and/or nitrate by catalysts or by ariy other means. The decrease of $\mathrm{Kjeldahl}$ -nitrogen above $260^{\circ} \mathrm{C}$ is due to either the denitrification or NOx formation.

PHOSPHORUS - Fig.3 shows the relation between the temperature and phosphorus output. The percentage of total and soluble(filterable) phosphorus In the oxidized liquor to the quantity of raw material versus the respective

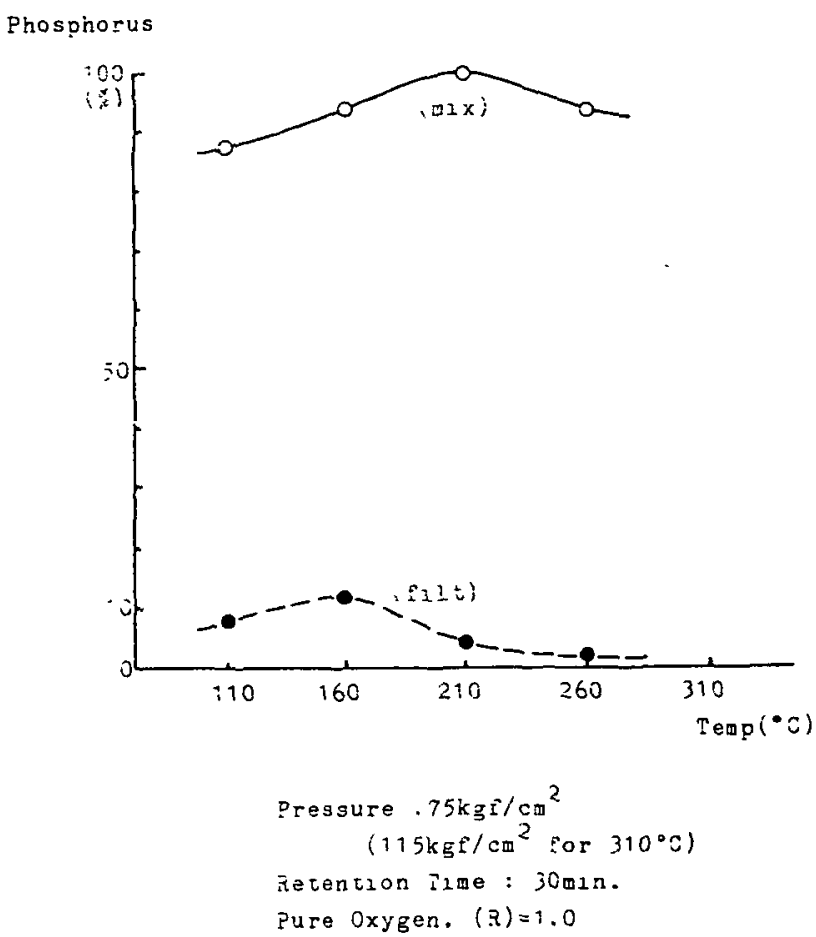

Fig.3 Reaction Temperature and Phosphorus

temperature is shown. The ratio of phosphorus in the liquid to the total phosphorus quantity is remarkably small comparing with the one of nitrogen in the liquid to the total nitrogen quantity, which gives only approximately 10 per cent at best. This may be caused by the fact that sewage sludge contains various cations such as calcuum ions which produce unsoluble salts with phosphorus. In CELSS, the wet oxidation should function to re-supply nutrient sources for plant culture in hydroponic solutions. It is rather desirable for nutritious elements such as phosphorus, nitrogen etc, to be dissolved in water. Therefore, this phenomenon 15 not favourable in making use of wet oxidation in CELSS. The behavior of phosphorus in wet oxidation has not been elucidated except in the case of sewage sludge. In this respect, the study of phosphorus is greatly needed.

\section{EFFECTS OF THE REACTION TIME}

CARBON - Fig.4 shows the status of TOC along with time. The reaction temperatures are $210^{\circ} \mathrm{C}$ nnd $260{ }^{\circ} \mathrm{C}$. In either cases, the percentage of $\operatorname{TOC}(\mathrm{mix})$ 
and TOC(filt) to the quantit) of a raw material is shown in relation with time. Pressure is kept $35 \mathrm{kgf} / \mathrm{cm}^{\wedge} 2$ in the case of $210^{\circ} \mathrm{C}$, and $75 \mathrm{~kg} f / \mathrm{cm}^{\wedge} 2$ in the case of $260^{\circ} \mathrm{C}$. The added oxygen index(R) is unity, and pure oxygen is used as a oxygen source. As this is a batch process, time is needed to reach the designated temperature. In order to know the changes if TOC in the heating process, the quaritity of TOC is checked at the temperature of $160^{\circ} \mathrm{C}$. The results are shown in the same figure.

The oxidation at $260{ }^{\circ} \mathrm{C}$ occurs markedly in the initial 15 minutes: But after that, the reaction curve declines - gradually. Fig.4 indicates that, oniy 80 per cent of TOC $(\mathrm{mix})$ is oxidized at 260 ${ }^{\circ} \mathrm{C}$, even if the reaction time exceeds 45 minutes. The solubilization has finished before. the temperature of the reactor " rearhes the designated temperature. As a result, TOC(filt) quantity will be the same as $\operatorname{TOC}(\mathrm{mix})$.

At $210^{\circ} \mathrm{C}$, only 15 per cent of TOC ( $m i x)$ is oxidized at zero minute on the ,horizontal axis of reaction time in of 19.4 . = The decrease of $\operatorname{TOC}\left(\mathrm{mIx}_{1}\right)$ is remarkable during the first 15 minutes, even if it does not show so rapid a decrease as seen at $260^{\circ} \mathrm{C}$. After that, the oxidation curve declines gradually. The solubilization of TOC reaches a plateau status after the 15-minute

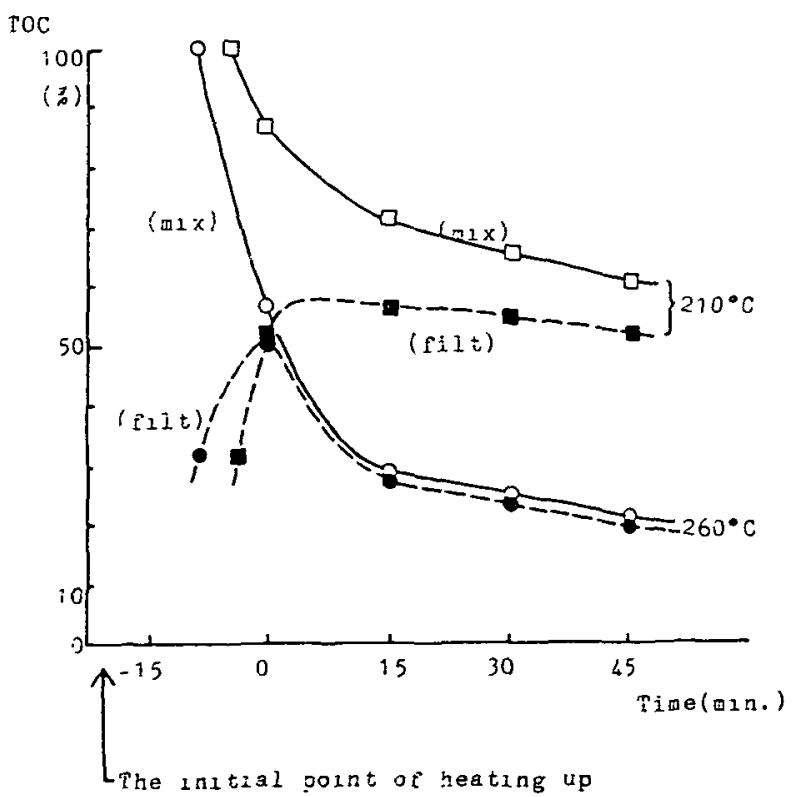

Fig.4 Reaction Time and TOC (1) process. But suspended TOC, i.e. TOC $(\mathrm{mix})$ miniss TOC (filt), does not disappear even gfter 45 minutes.

$\operatorname{TOC}\left(\mathrm{m}_{1} x\right), \operatorname{TOC}(f i \mid t)$ and the converted carbon quantities of acetic acid and $C_{2}-$ C6. irgaric acids are shown in fig.5 in relation to their percentage to raw materials versus a time. The reaction temperature in this case $15260^{\circ} \mathrm{C}$. The other experimental conditions are the saine as in fig.4. As it was explained previously, the solubilization of TOC has finished before the reaction temperature reaches the designated temperature. $C_{2}-C_{6}$ organic acids reach their maximum within

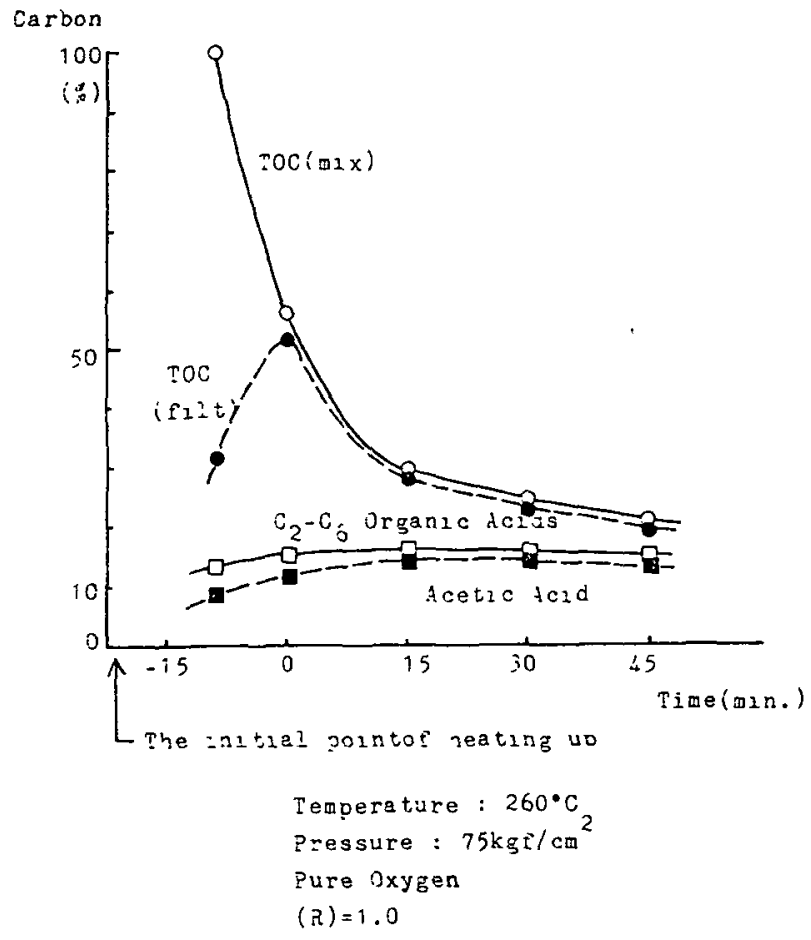

'Fig.5 Reaction Time and TOC (2)

15 minutes and do not decrease any more despite that $\operatorname{TOC}\left(\mathrm{m}_{1} \mathrm{x}\right)$ decreases. The ratio of acetic acid to $\mathrm{C}_{2}-\mathrm{C}_{6}$ organic acids increases with time until 30 minutes but does not decrease even after 45 minutes.

Fig. 4 and 5 show that the reaction temperature determines the upper 1 imit of the oxidation. Considering the results of fig. 1 together with $\mathrm{fig.4}$ and 5 , it is understood that only 80 per cent of organic compounds in raw materials is oxidized even at the reaction temperature of $310^{\circ} \mathrm{C}$, and the remaining 20 per cent is non-combustible and consists mainly of 
acetic acid.

Probably, acetic acidwill not be oxidized at more than $310^{\circ} \mathrm{C}$. In CELSS, it is desirable that organic compounds in a raw material are wet-oxidized

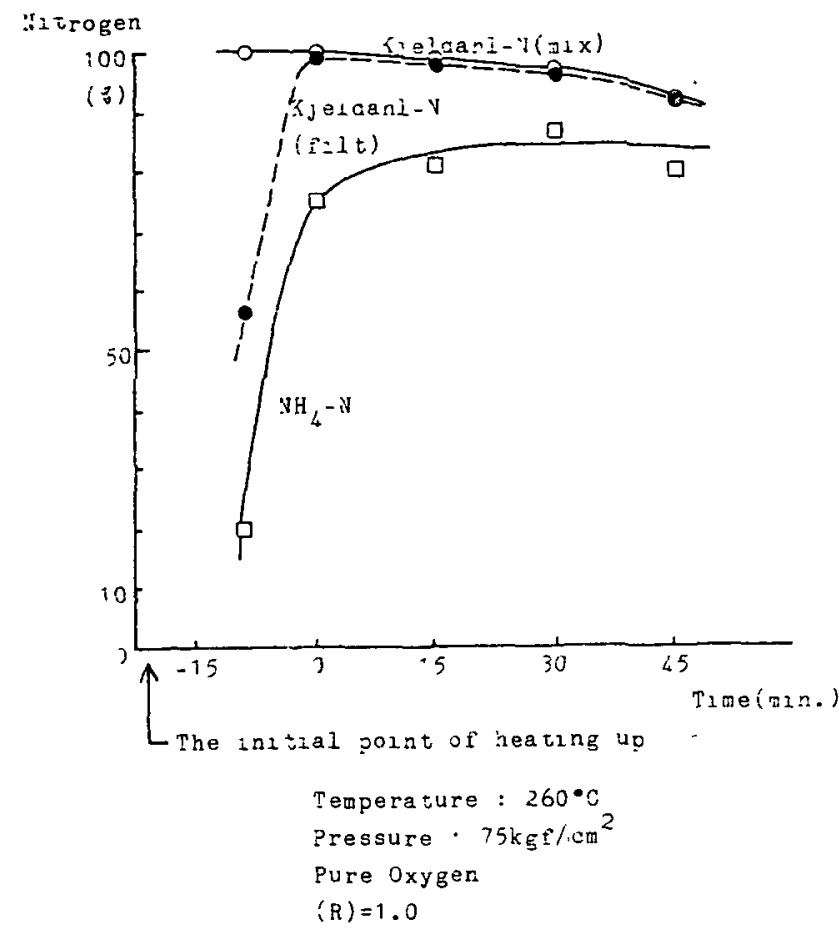

Fig.6 Reaction Time and Nitrogen

comisletely, and then it is necessary to develop the catalysts which have capability to oxidize acetic acid completely or to change the course of the reaction of wet-oxidation without producing acetic acid.

Nitrogen - Fig.6 shows the status of the nitrogen over time. The conditions in $\mathrm{fig}_{19} \mathrm{are}$ the same as the ones of in fig. 5 experimentally and materially. The solubilization of the suspended nitrogen compounds has finished before the reactor has reached the designated temperature. The dissolved nitrogen compounds has led to ammonia in the same time. After the designated temperature has been realized, the Kjeldahl-nitrogen curve declines gradually with time. The percentage of ammonia to the $K j e l d a h l-n i t r o g e n$ forms the plateau status after $15 \mathrm{minutes}$.

TIME-COURSE OF VARIOUS KINDS OF MATERIALS

Fig.7 shows the time-course of "arious kinds of materials: in wet oxidation. Each material is wet-oxidized urider the conditions of $260^{\circ} \mathrm{C}$ and $75 \mathrm{kgficm} 2$. Pure oxygen is used as an o.yger, source. As discussed previously, acetic acid is virtually non-combustible, so orly a few percent of acetic acid are oxidlzed. About 50 per cent of stearic arid is oxidized in an hour. Casein, sewage sludge and cellulose show similar shararteristics to one another in the time-course curves. It can be easily revolized by their positions in the figure that formic acid and starch are $\rightarrow$ easily oxidized.

It is also known that cyanates in raw materials are oxidized to ammonia at 100 fer cent, and 0.01 per cent of it remains iri gas. Sulfur, sulfide' or sulfite, is oxidized to sulfate and remains in liquid. Sodium, potassium and -hlorine lons are intact under the wet oxi.dation, and remain in liquid.

There are various kinds of materials, some are easy to oxidized, and.some are rot. The research of wet oxidation in the CELSS project should start based on ithe elabolate choice of suitable raw materials.

In CELSS, raw materials will be

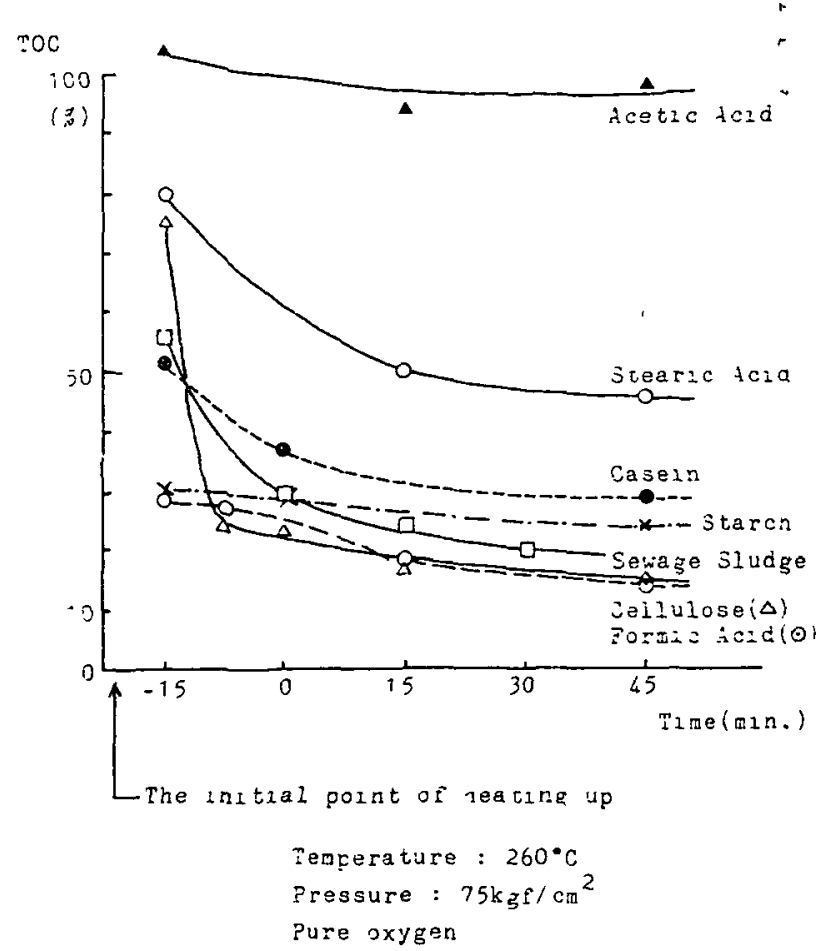

Fig.7 Time-course of Various Kinds of Materials 
kitchien and laundry wastewater, feces, non-edible parts of plants, used hydroponic solution, trash and so on. They are entirely different from sewage sludge in character. Although the results of wet oxidation studies of sewage sludge carnot be apslied directly to CELSS, because thie fundamental phenomena are the same, the results of wet oxidation studies of sewage sludge can be of reference to the studies of CELSS.

\section{BEHAVIDRS OF METALS}

Thie author has not yet studied the behaviour of metals in wet oxidation experimentally, but has done so in the field. The studies of a wet oxidation facility of sewage sludge in the Yok:ohama north-side-sewage-treatment plant, whose operating conditions are $240{ }^{\circ} \mathrm{C}$ in temperature, $72 \mathrm{kgf} / \mathrm{cm}^{\wedge} 2$ in pressure and 1 hour in retention time, show that most of $\mathrm{Cd}, \mathrm{Zn}, \mathrm{Cu}, \mathrm{Pb}, \mathrm{Cr}, \mathrm{Mn}$ or $\mathrm{Fe}$ contents in a raw material are transferred to solid after the wet oxidation.

One man, even in a normal life, usually uptakes daily 0.05, 10.9, 5.85, $2.77,0.177,0.354,0.022 \mathrm{mg}$ of $\mathrm{Cd}, \mathrm{Zn}$, $\mathrm{Mn}, \mathrm{Cu}, \mathrm{Pb}$, As, Hg respectively. These are excreted and urinated daily at 1000 to $1200 \mathrm{mls}$. It is unknown how much part of the respective element will be in 1 iquid or solld after the wet oxidation. Therefore, trial oxidation in the CELSS project should be carried out after the studies of the metal behaviors have been established.

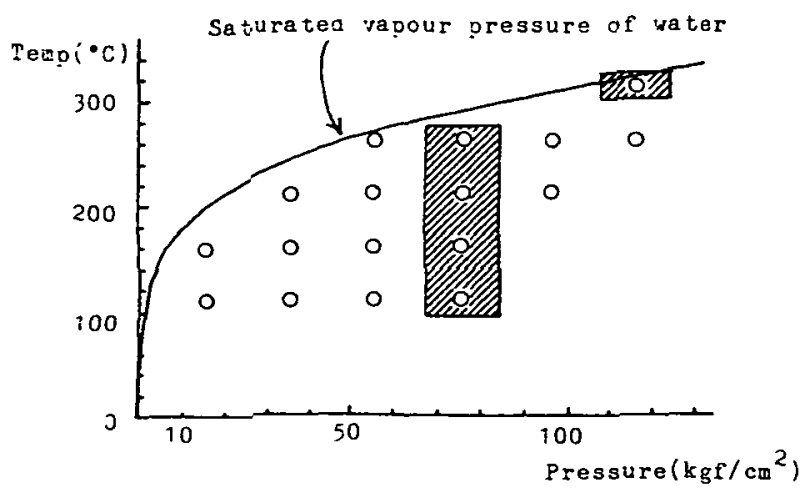

Fig.8 Saturated Vapour Pressure of Water
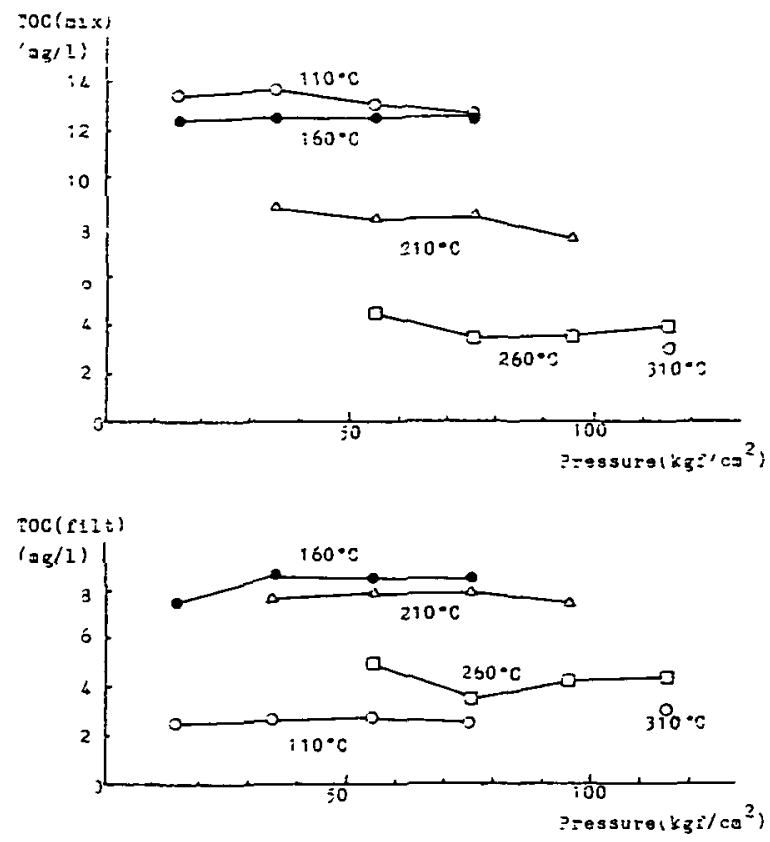

Fig.9 Pressure and 0xidized Ratio

\section{PRESSURE AND OXIDIZED RATIO}

Reaction pressure of the wet oxidation is determined as the sum of saturated vapor pressure of water at a certain reaction temperature and the pressure of gases which were introduced into the reactor at room temperature before the beginning of the experiment.

Fig.8 shows a curve of a saturated vapor pressure of water. A reaction cannot take place in the conditions of any temperature-pressure combination above the curve. Experiments to study pressure effects are carried out under the conditions of temperature-pressure combinations below the curve shown in the figure $(*)$. The reaction time is 30 minutes and the added oxygen index(R) is unity. The results of tests executed under these combinations of condition are shown in fig.9.

Fig.9 shows that the oxidized ratio depends on the reaction temperature only. This fact is true also in the case in which a mixed gas of oxygen and $n i t r o g e n$ is used as an oxygen source. It means that a pure oxygen gas is not a necessary material as an oxygen source in the wet

(*) The experimental conditions adopted in fig.1-3 are the ones enclosed by the hatched area in this figure. 
sxidation. In other words, the essential fact is to supply a necessary quantity of oxygen in either pure or mixed status. This fact may be favorable to CELSS.

\section{EFFECTS DF CATALYSTS}

As discussed earlier, organic compounds in raw materials can not be oxidized completely and nitrogen compourids remain in liquid in the form of ammonia in the wet oxidation. If and when a wet oxidation facility is used in CELSS, it is desirable that carbon in organic materials shall be involved in a gas recycling system in a form of carbon dioxides and that nitroger, ir them is transformed to plant-avallable nitrates, by completirig the oxidation process. In order to realize tre above process in a short time without by-products, it is supposed that there would be no alternative way besides utilizing certain catalysts for the time being.

From a sewage-sludge-treatment point of view, the authors have been engaged in research to develop the catalysts so that the wet-oxidation system can exhibit a similar function to a usual combustion furnace system, and remove nitrogen from 1 iquid.

As the result of surveys, it has become clear that noble metals such as Pt, Fd, Fin and Ru among the transition elements are hopeful as catalysts. These metals are needed to be supported on carriers made of alumina and titania etc.

To prevent suspended particles in 1 iquid from poisoning a catalyst, filtrated-wet-oxidized-sewage-sludge cutput is used in the wet oxidation tests on the catalysts. One catalyst has been used 20 times in wet oxidation of fresh "filtrates. The results are as follows.

When a Pt or Pd catalyst is used, nitrogen in the $l$ iquid is denitrified at 100 per cent. When a Ru or Rh catalyst is used, a half of nitrogen in the liquid is denitrified. The rest remains in the $l$ iquid as a nitrate when Ru is used and as ammonia when $R h$ is.

On the other hand, organic compounds are not catalytically oxidized at all, when $P t$ is used. The wet-oxidized output of TOC decreases to a half of a control, when $\mathrm{Rh}$ is used. The oxidation chalysis
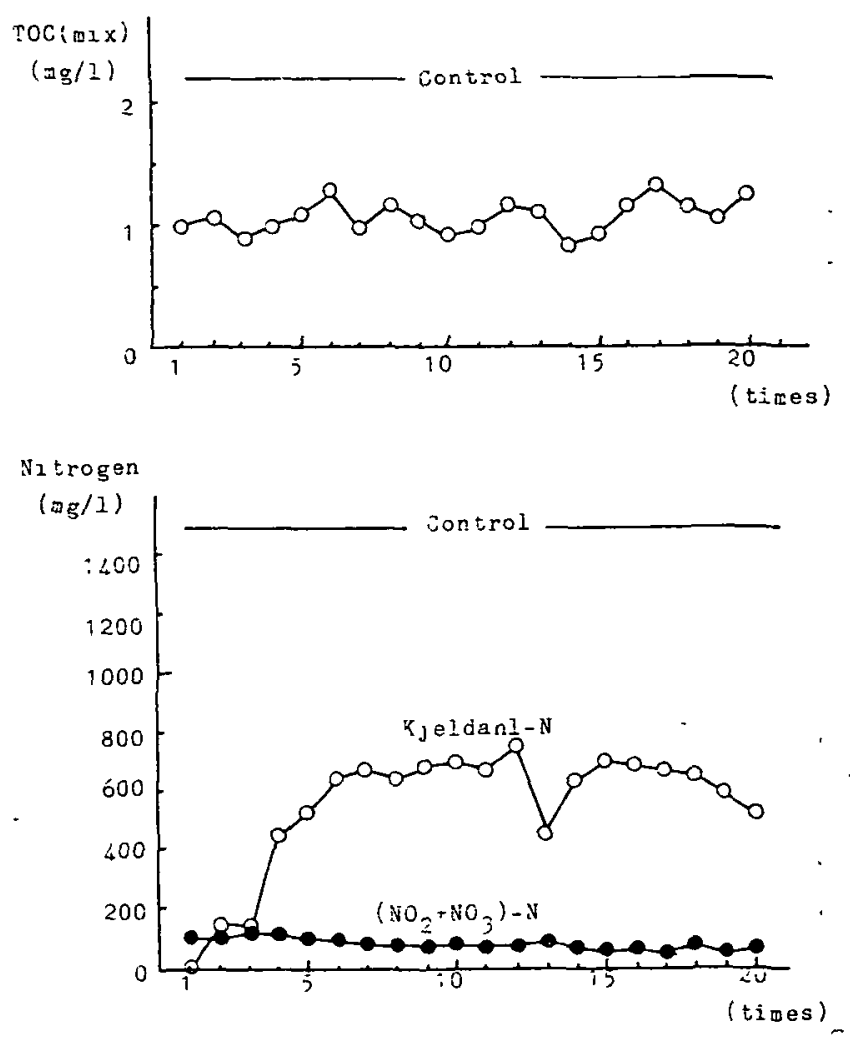

Fig.10 Effects of Rh Catalyst

of Ru or Pd 25 better than that of $P_{t}$ and worse than that of Rh. The result of $R h$ is shown in fig. 10 .

Good catalysts for both oxidation and $n$ itrification have not yet been found up to now. Therefore, research should be initiated to develop the catalysts which shall be involved in a recycling system of CELSS, will not be poisonous, to working staff as well as living things in a space station, and shall have a effective catalysis for oxidation and nitrification.

\section{CONCLUSIONS}

The behaviors of various kinds of materials in wet oxidation were shown and the applicability of wet oxidation to CELSS was discussed. In Japan, a wetoxidation facility for CELSS has been designed already. In this regard, the following projects are to start promptly:

(1)To manufacture a trial wet-oxidation facility,

(2) To execute tests of wet-oxidation for selected materials which are expected to be used in practice, and

(3) To observe the behavior of the 
materials.

The complete solubilization of raw inaterials will be a desirable way from the element-redistribution point of view. On the other hand, $2 n$ the case of unsoluble phosphorous compounds, there would be no iroblem if they should be sprinkled to a plant culture in a hydrofionic solution, and, at the same time, if the plant culture would be able to utilize them as nourishment.

In this respect, studies and discussions to confirm if each element, in a wet-oxidation output, is desirable in a liquid, solid or a gas phase. Thus, further studies on chemical-form contrrlable catalysts of each element can commerice effectively.

\section{Acknowledgement}

The authors express their appreciation to Dr. S. Matsuno for his review and comment. 